\section{A Change in Microbial Virulence Under Simulated Microgravity Might Hold a Strategic Value for Salmonella}

\author{
Ajay Nair* \\ Oklahoma Medical Research Foundation, Oklahoma City, USA
}

\begin{abstract}
The extensive research done on microbes in space, especially experimenting the impact simulated gravity has on the growth and sustenance of microbes, has improved our understanding on the mechanisms of pathogenesis and host-pathogen interactions. In the recent past, studies have shown that an underlying change in gene activity manifests in the form of differential responsiveness to weightlessness among microbes. Between the various microbes examined, a common food borne pathogen Salmonella becomes more virulent when grown in simulated microgravity. Many theories have emerged to explain this phenomenon. This review will not only summarize some of the key research done on microbial virulence under virtual gravity, but also provide a new hypothesis to explain how a state of enhanced virulence might help microbes adapt in microgravity.
\end{abstract}

Keywords: Adaptation; Microgravity; Pathogenesis; Salmonella; Virulence

\section{Introduction}

In the struggle for continuity, the difference between success and failure is not luck but better adaptation. Natural selection acts on gradual changes in phenotype due to shift in the genotype of a group in a population. These phenotypic variants ensure that the most successful individuals are those that have traits better suited to adapt to their immediate environment. However, no matter how drastic or sudden, a change in the environment does not bring a change in genetics. Such changes add constraint, by way of increased selective pressure, on those few genetic variants that get favored by natural selection. The basic progression from the simplest to the most complex has been in fact an ending of millions and millions of years of natural selection. Though, this transformation was and never will be predestined. It so happens that a few among a population always showed better adaptation with the changing environment;

*Corresponding author: Ajay Nair, Oklahoma Medical Research Foundation, Oklahoma City, USA, Tel: +1 4059856960; E-mail: ajay-nair@omrf.org

Citation: Nair A (2015) A Change in Microbial Virulence Under Simulated Microgravity Might Hold a Strategic Value for Salmonella. J Infect Non Infect Dis 1: 009.

Received: October 08, 2015; Accepted: November 12, 2015; Published: November 26, 2015 additionally, an error or mutation in their genetic makeup gave them the impetus to survive and their lineages gave birth to a race tougher and better equipped than its ancestry; what we call as being evolutionarily complex.

The effect of Modeled Microgravity (MMG) on cellular reactions has been primarily explored by the use of a specialized bioreactor called the High Aspect Ratio rotating wall Vessel bioreactor (HARV; Synthecon Inc., Houston, Tex.). HARV creates simulated weightlessness by altering the gravitational vectors over the surface of cells (Figure 1). This results in an over-all-time-averaged gravitational vector of $10^{-2} \mathrm{xg}$, as discussed by Unsworth and Dedolph $[1,2]$. Gravity as low as this produces a sustained low shear milieu for cell growth, one that is suited for all experiments analyzing the effects of microgravity.

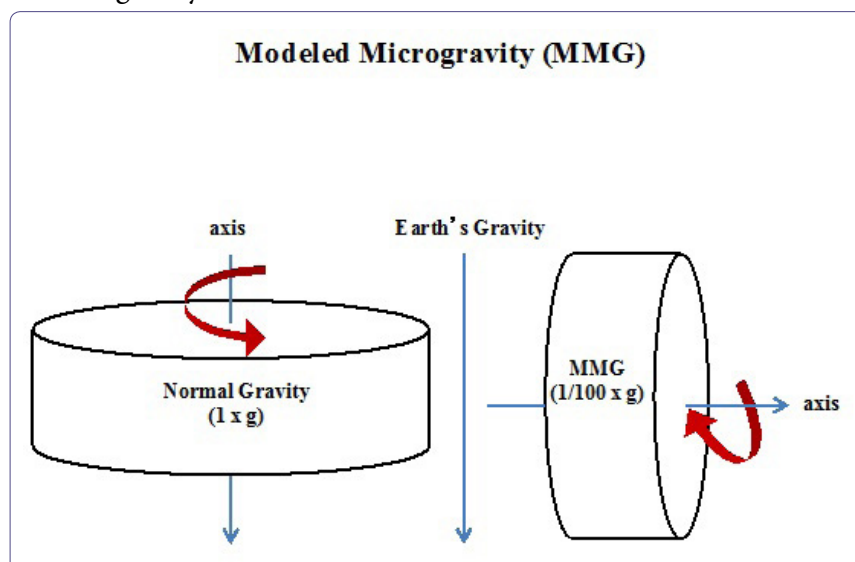

Figure 1: Principle behind Modeled Microgravity (MMG) in HARV.

Left - Bioreactor vessel with its axis of rotation parallel to earth's gravity, offers normal gravity $(1 \mathrm{xg})$

Right - Bioreactor vessel in MMG orientation, with its rotational axis perpendicular to the gravity nullifies the downward gravity vector $(10-2 \times g)$ [3]

\section{Microbes in Space, A Bad Idea}

The effects of microgravity on microbial growth and life processes, such as virulence, have been investigated in the recent past. In order to examine the aforesaid parameters, several ground-based models have been developed, in particular the HARV. One of the Classic studies suggests that Salmonella enterica serovar Typhimurium exhibited increased virulence under conditions of simulated microgravity, as discussed by Nickerson, Rozenweig and Sarker [3-5]. Parallel to this work was the one, which strived to reason out the causes for differences in growth pattern seen in yeast cultures when subjected to simulated microgravity, as discussed by Johanson [6]. Such an alteration to microbial virulence has also been validated with a corresponding change in gene expression. But in spite of all the mounting evidence, one could argue as to why was this particular species of Salmonella selected amidst so many other pathogens? And the answer to this comes from few interesting findings from the past. Firstly, environmental influences alter the gene expression of virulence determinants in pathogenic bacteria. Salmonella spp., in particular, seem to be quite sensitive to changes in $\mathrm{pH}$, osmolarity, stress and growth phase, as discussed by Foster and 

009.

Mahan [7,8]. Secondly, the National Center for Infectious Diseases claims that Salmonella enterica serovar Typhimurium is the leading cause for systemic diseases in humans, especially in immune-compromised individuals. Now in correlation, several studies have also concluded that spaceflight dampens the immune response of the body, as discussed by Nefedov, Pellis and Taylor [9-11]. So it is clear that astronauts, during their space travel, are no better than patients with blunted immune systems and therefore, an extensive examination of this bacterium under modeled microgravity is of utmost significance.

Experiments using Low Shear Modeled Microgravity (LSMMG) have been shown to induce differential gene expression in Salmonella enterica serovar Typhimurium. Changes included those in the expression of transcriptional regulators, virulence factors, biomolecule-biosynthetic enzymes and iron utilization enzymes, as discussed by Rozenweig [4]. Amid numerous results, a few stood out quite distinctively in demonstrating the effect of microgravity on microbial virulence. The oral lethal dose to kill $50 \%$ of animals, which is also termed as the $\mathrm{LD}_{50}$, for Salmonella enterica serovar Typhimurium, under modeled microgravity was significantly lower (roughly 5.2 times) than those grown under normal conditions; 4.3 $\mathrm{x} 10^{6}$ as opposed to $2.2 \times 10^{7} \mathrm{CFU}$ respectively. In addition, a survival assay using mice revealed that the time of death post-infection with the specific Salmonella spp., which were grown under simulated states, decreased considerably (Figure 2).

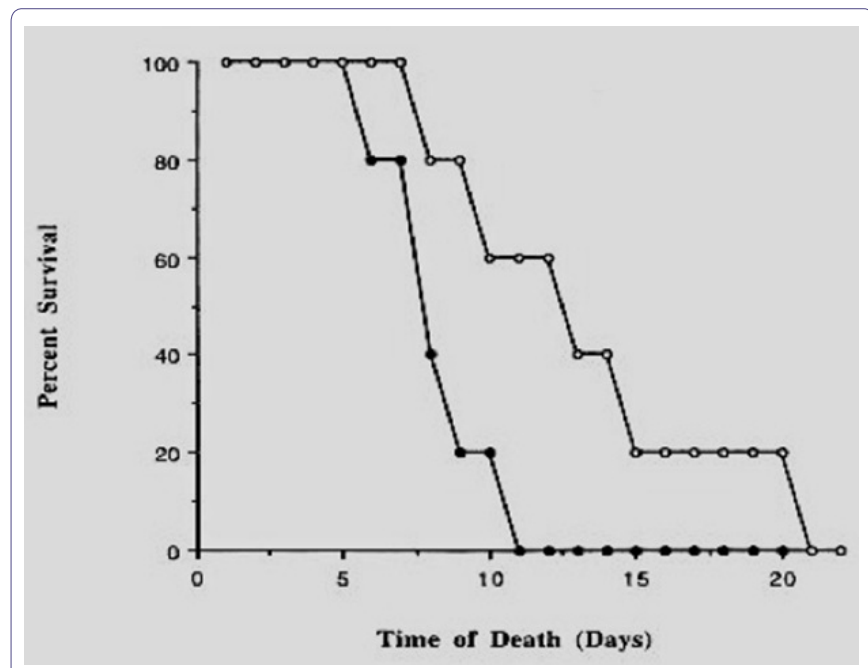

Figure 2: Survival of mice after oral infection with serovar Typhimurium 3339 grown under MMG or normal gravity.

Serovar Typhimurium 3339 grown under MMG (black dots) or normal gravity (white dots) was administered perorally as individual infections to 8-week-old female BALB/c mice at inoculum titers of $1.9 \times 106$ and $1.9 \times 106 \mathrm{CFU}$, respectively. The percent survival is defined as the percentage of mice infected with MMG- or normal-gravity-grown 3339 organisms surviving at the indicated number of days post-infection [3].

Also, results from acid resistance assays revealed that MMG grown bacteria were three times more resistant to low $\mathrm{pH}$ than the control strains, as discussed by Nickerson [3] (Figure 3). So a part of an MMG grown bacterium's enhanced virulence might be due to their resilience towards acidic stress during the microbe's passage through the host's stomach. The latter being one of the prime non-specific defenses towards foreign invasion.

Acid resistance defect in S. Typhimurium ydcI mutant. Isogenic WT and ydcI S. Typhimurium strains were tested for resistance to osmotic $(2.5 \mathrm{M} \mathrm{NaCl})$, thermal $\left(50^{\circ} \mathrm{C}\right)$, and acid ( $\mathrm{pH} 3.5$ using citric acid or $\mathrm{HCl}$ ) stress over a time period of $120 \mathrm{~min}$. The percent survival

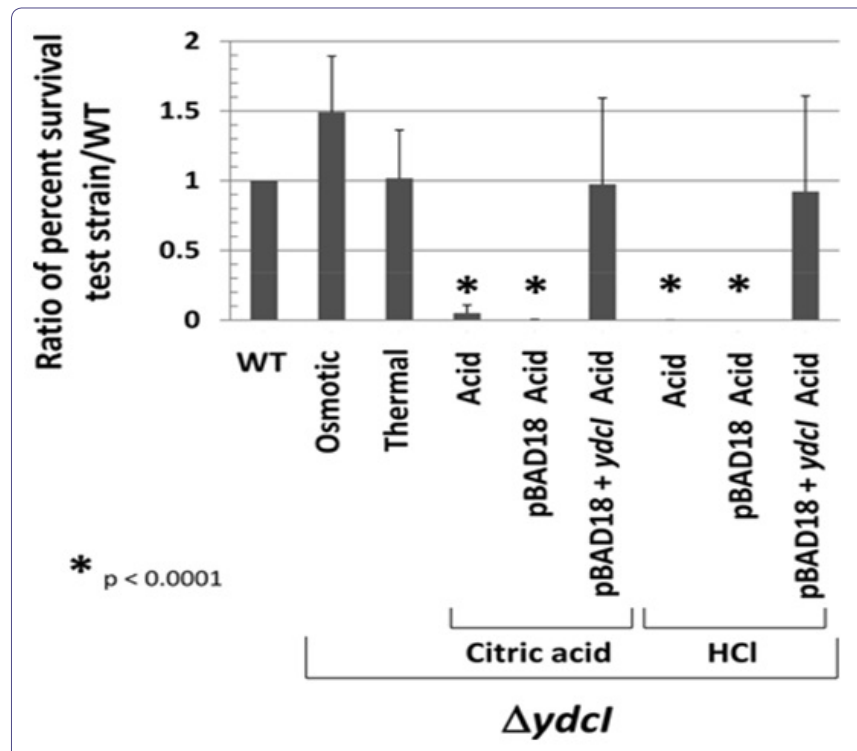

Figure 3: Acid resistance assay.

of the WT and ydcI mutant strains under the corresponding stress (compared to before addition of stress) was calculated, and a ratio of the percent survival of the indicated ydcI mutant test strain to that of the WT strain is shown. The ydcI mutant strain containing pBAD18 or $\mathrm{pBAD} 18+\mathrm{ydcI}$ was grown in the presence of arabinose [12].

To determine how LMMG cultured cells respond to macrophage killing, the intracellular survival capacities of MMG grown salmonella within murine macrophage-like cell lines was analysed. In contrast to the standard cultures, a surprising 81 -fold increase in the survival was observed in the first twenty minutes after infection, as discussed by Nickerson and Ralph $[3,13]$. To corroborate results from Salmonella, a few other pathogens were subjected to LMMG assays. Simulated weightlessness affected the virulence of Staphylococcusaureus by making the strains produce additional hemolysins (for e.g. $\alpha, \beta$ and $\gamma$-toxins), as discussed by Rosado [14]. Not only this, microgravity exposed bacteria seemed to be better equipped in dealing with others stressors like: osmolarity, $\mathrm{pH}$, temperature etc. Biofilm production is also an important indicator of microbial virulence. Inside a biofilm, bacteria display superior resistance to stresses like oxidative, osmolarity, $\mathrm{pH}$ and antibiotic. An experiment that involved subjecting LMMG exposed biofilm producing $E$. coli to two stressors- ethanol and penicillin resulted in the production of thicker biofilms than their control counterparts, as discussed by Lynch and Benoit [15-17].

Following these intriguing results, several works have been carried out to establish relationships at the genetic level. Proteins (e.g. $y d c I$ ) of the class transcriptional regulators have been found to be one of the prime low fluid shear stimulon (as described using microarray analysis). The gene $y d c I$ has been, in particular, studied quite extensively with reference to MMG grown Salmonella enterica serovar Typhimurium. This gene seems to be (i) conserved across genera, as discussed by Jennings [12]. (ii) appears to be a member of the rpoS gene regulon (biofilm production in pathogenic bacteria is controlled by the rpoS gene encoded product $\sigma^{s}$, as discussed by Jennings and Adams [12,18], (iii) is associated with bacterial acid resistance, (iv) upregulated significantly by low fluid shear growth conditions, as discussed by Wilson [19,20] (Figure 4). 


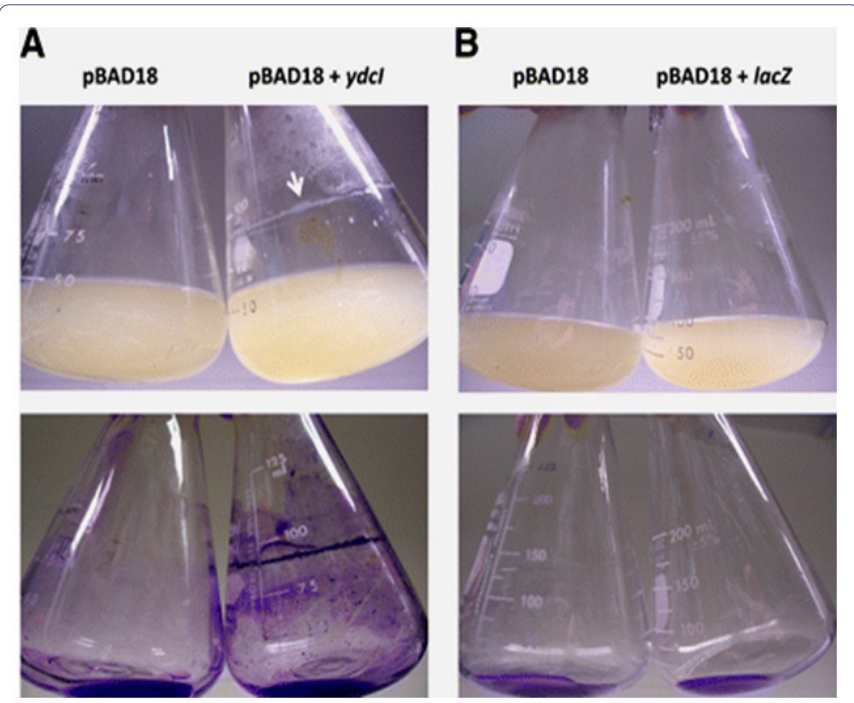

Figure 4: Induction of ydcl expression results in S. Typhimurium biofilm formation.

(A) S. Typhimurium strain 3339 containing either pBAD18 or pBAD18+ydcl was grown in the presence of arabinose for approximately $16 \mathrm{~h}$ in LB medium. The white arrow indicates significant biofilm accumulation at the medium-air interface. The upper and lower panels show culture appearance and crystal violet-stained flasks (with cultures removed), respectively;

(B) The same as panel A, except that strain 3339 containing pBAD18+lacZ was tested

Despite ground-breaking results, none of the above-mentioned substantiations have concentrated on reasoning out the advantage a microbe may hold by an augmented virulence under conditions of microgravity. Hence, the following parts of the review will present a notion that will attempt to rationalize this puzzling behavior displayed by pathogenic bacteria.

\section{Enhanced Virulence Under Microgravity is Important to Microbial Perpetuation}

Virulence is the degree of pathogenicity of a microbe to cause a disease. But this term implies to us and not to the microbe. For a microbe this is one of the potent tools for its own survival and defense. Disease is, in a way, an after effect of a microbe's habitation, for its further continued existence inside the host. Hence, virulence has to be explained here from a different perspective. The eventual want of a microbe, during its occupancy, is the host's survival as long as possible for its own endurance. Consequentially a chronic infection causing- pathogen must be considered evolutionarily more significant than an acute infection causing-pathogen because the former assures the microbe with a longer survival in the host and hence is beneficial. The advantage that a microbe may hold by enhanced virulence in microgravity, may well be explained by considering two possibilities.

1. Micro gravity in a way presents an adverse environment. This changed state of weightlessness may influence underlying gene expression pathways among genetic variants in the population. Mutation has been the very difference between success and failure in the struggle for survival. It provides variations in populations and only those with beneficial variations tend on average to survive and become ever more specialized, to suit the requirement. As the mutant strains divide, more and more number of altered strains are formed at subsequent generations and resultantly this diverged lineage is naturally selected, as being fit to survive under weightlessness.
2. The enhanced virulence shown by the transmuted strains may hold a strategic role in space for the microbe. Now we know that energy is consumed for two central processes in a living system. One is for Reproduction (R) and the other for Maintenance (M) (Figure 5A). Under usual circumstances, the balance between the two is upheld for the perpetuation of life. During favorable times, Energy (E) is abundant in the system. And this leads to increase in the net entropy, the degree of disorderness. To circumvent this, energy is equally distributed between the two processes. An additional factor that we are familiar with is that in strains of high virulence only few cells are sufficient to infect and cause the disease, as discussed by Pelzar and Zotin [21,22]. Micro gravity and the other cultural necessities in space are completely in optimum and unfavorable in comparison to that on earth. Genetic variants within the microbial population would, momentarily, opt for enhanced virulence with the sole intention that less number of cells may be employed to infect. This would in turn help reserve much of the cells free energy for cell maintenance rather than using them for producing more number of cells (via reproduction). On the contrary, if energy is equally dispersed (Figure 5B), the population will not only produce progeny with virulence levels below the minimum threshold, but might also jeopardize the sustenance of the entire population, owing to insufficient energy both for cell maintenance and reproduction.

\section{Conclusion}

In the recent past, plasticity (the ability of an organism to change its phenotype in response to changes in the environment) has been recognized as a positive driving force of evolution that sets the stage for neo-Darwinian evolution by increasing the survival of those who display a plastic response in a population. This genetic variation can be selected upon so that evolution progresses in the direction of the induced plastic response, as discussed by Baldwin [23]. Waddington's genetic assimilation, as discussed by Waddington [24] is based on the premise that environmentally triggered phenocopies (plastic responses) may become genetically fixed in the population via repeated selection. Some of the recurring themes that mark the theoretical discussions of genetic assimilation include: (1) presence of a plastic response in the beginning to start the process; (2) the possible role for epigenetic mechanisms in the process; (3) the limits imposed by costs of plasticity; (4) the apparent quickness of the process in comparison to standard evolution. Therefore, it is quite possible that a state of enhanced virulence exhibited by Salmonella spp. could be an acquired phenomenon in response to the simulated microgravity. And that an epigenetic mechanism could be underpinning the acquired inheritance of boosted virulence. After all, it is well known that bacteria, via a process called phenotypic variation, alter their pathogenicity in response to changing environments. And that this phenotypic heterogeneity can arise from mechanisms both genetic and epigenetic, as discussed by Turner [25]. DNA methylation has been known to control the phase-variable expression of virulence genes. A detailed explanation on the mechanisms is beyond the scope of this review.

Whatsoever is shaped in nature has to unquestionably put up with the elemental law- evolve or perish. Nature is an arena of pressure, and unless successful variations are acquired by organisms, the chances of their survival become very small. So an improved virulent form of the ground-based pathogens (e.g. Salmonella spp.) in space might be a bad 
Citation: Nair A (2015) A Change in Microbial Virulence Under Simulated Microgravity Might Hold a Strategic Value for Salmonella. J Infect Non Infect Dis 1: 009.

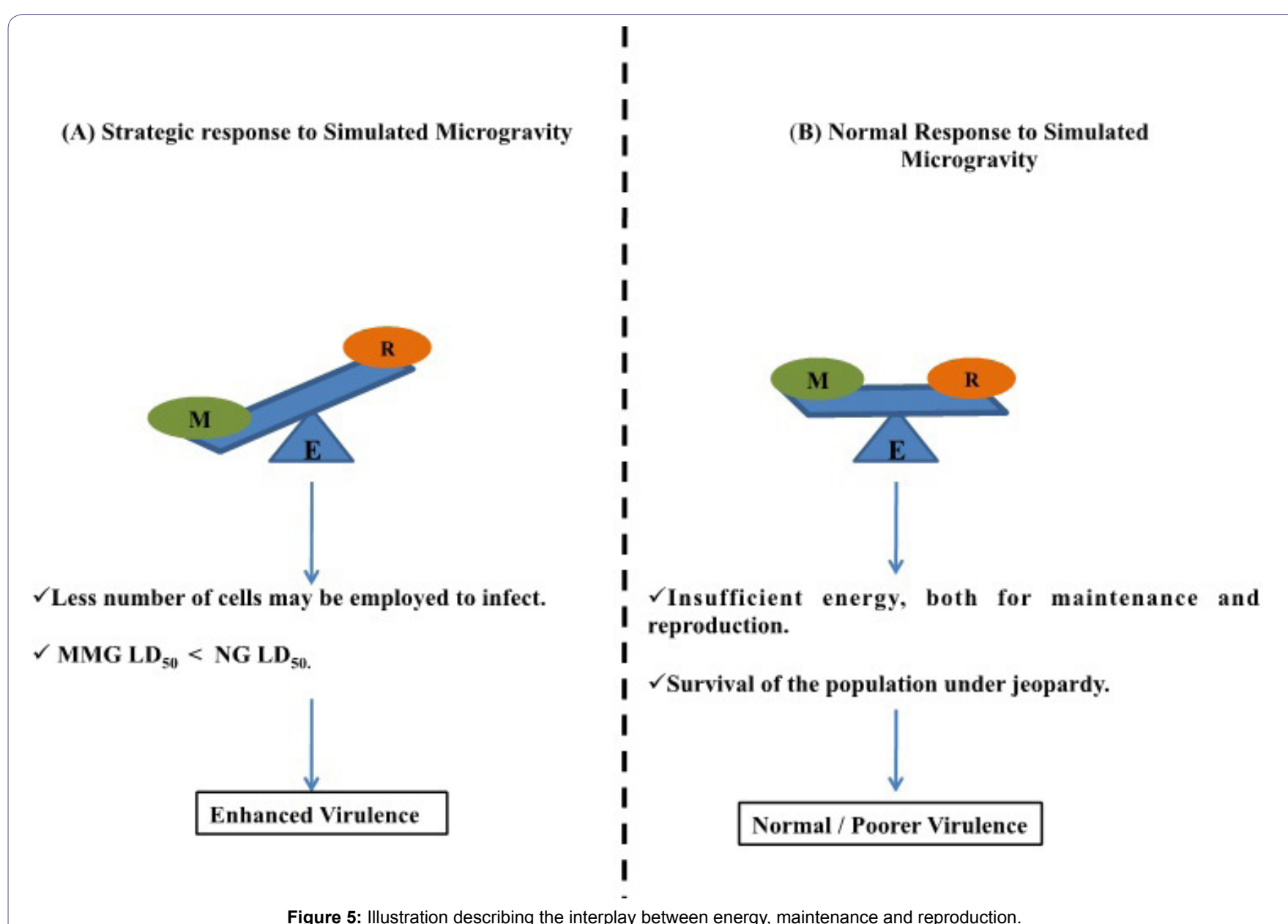

(A) Strategic response: owing to environmental stress, the energy balance between cell maintenance and reproduction is tipped; more energy is directed towards maintenance. As a result, less number of cells produced, and are therefore equipped with enhanced virulence, evident from the reduction in LD50, in comparison to the standard LD50. (B) Normal response: despite inoptimum conditions, the thermodynamic poise is maintained, resulting in microbes with normal or poorer virulence; putting the survival of the population at risk.

news on the pathological front. But it certainly benefits the microbe towards tiding over the adverse conditions created by altered gravity.

\section{References}

1. Unsworth BR, Lelkes PI (1998) Growing tissues in microgravity. Nat Med 4 901-907.

2. Dedolph RR, Dipert MH (1971) The physical basis of gravity stimulus nullification by clinostat rotation. Plant Physiol 47: 756-764.

3. Nickerson CA, Ott CM, Mister SJ, Morrow BJ, Burns-Keliher L, et al. (2000) Microgravity as a novel environmental signal affecting Salmonella enterica serovar Typhimurium virulence. Infect Immun 68: 3147-3152.

4. Rosenzweig JA, Abogunde O, Thomas K, Lawal A, Nguyen YU, et al. (2010) Spaceflight and modeled microgravity effects on microbial growth and virulence. Appl Microbiol Biotechnol 85: 885-891.

5. Sarker S, Ott CM, Barrila J, Nickerson CA (2010) Discovery of spaceflight-regulated virulence mechanisms in Salmonella and other microbial pathogens: novel approaches to commercial vaccine development. Gravitational and Space biology 23: 2 .

6. Johanson K, Allen PL, Lewis F, Cubano LA, Hyman LE, et al. (2002) Saccharomyces cerevisiae gene expression changes during rotating wall vessel suspension culture. J Appl Physiol (1985) 93: 2171-2180.

7. Foster JW, Spector MP (1995) How Salmonella survive against the odds Annu Rev Microbiol 49: 145-174.
8. Mahan MJ, Slauch JM, Mekalanos JJ (1996) Environmental regulation of virulence gene expression in Escherichia, Salmonella, and shigella spp. Escherichia coli and Salmonella cellular and molecular biology. ( $\left.2^{\text {nd }} e d n\right)$, ASM Press, Washington DC, USA. Pg: 2803-2816.

9. Konstantinova IV, Nefedov luG, Eremin AV, Drozdova VI, Skriabin AS (1978) Immunological reactivity and prediction of allergic complications in the crew of the second expedition of Salyut 4. Kosmicheskaia Biologiia i Aviakosmicheskaia Meditsina 12: 15-19.

10. Pellis NR, Goodwin TJ, Risin D, Mclntyre BW, Pizzini RP, et al. (1997) Changes in gravity inhibit lymphocyte locomotion through type I collagen. In Vitro Cell Dev Biol Anim 33: 398-405.

11. Taylor GR (1974) Space microbiology. Annu Rev Microbiol 28: 121-137.

12. Jennings ME, Quick LN, Soni A, Davis RR, Crosby K, et al. (2011) Characterization of the Salmonella enterica serovar Typhimurium ydcl gene, which encodes a conserved DNA binding protein required for full acid stress resistance. J Bacteriol 193: 2208-2217.

13. Ralph P, Nakoinz I (1975) Phagocytosis and cytolysis by a macrophage tumour and its cloned cell line. Nature 257: 393-394.

14. Rosado H, Doyle M, Hinds J, Taylor PW (2010) Low-shear modeled microgravity alters expression of virulence determinants of Staphylococcusaureus. Acta Astronautica 66: 408-413.

15. Lynch SV, Mukundakrishnan K, Benoit MR, Ayyaswamy PS, Matin A (2006) Escherichia coli Biofilms Formed under Low-Shear Modeled Microgravity in a Ground-Based System. Appl Environ Microbiol 72: 7701-7710. 
16. Benoit M, Klaus D (2005) Can genetically modified Escherichia coli with neutral buoyancy induced by gas vesicles be used as an alternative method to clinorotation for microgravity studies? Microbiology 151: 69-74.

17. Benoit MR, Li W, Stodieck LS, Lam KS, Winther CL, et al. (2006) Microbial antibiotic production aboard the International Space Station. Appl Microbio Biotechnol 70: 403-411.

18. Adams JL, McLean RJ (1999) Impact of rpoS deletion on Escherichia coli biofilms. Appl Environ Microbiol 65: 4285-4287.

19. Wilson JW, Ott CM, Ramamurthy R, Porwollik S, McClelland M, et al. (2002) Low-Shear modeled microgravity alters the Salmonella enterica serovar typhimurium stress response in an rpoS-independent manner. Appl Environ Microbiol 68: 5408-5416.

20. Wilson JW, Ramamurthy R, Porwollik S, McClelland M, Hammond T, et al. (2002) Microarray analysis identifies Salmonella genes belonging to the low shear modeled microgravity regulon. Proceedings of the National Academy of Sciences USA 99: 13807-13812.
21. Pelzar MJ Jr, Chan ECS, Krieg NR (1986) Microbiology. ( $5^{\text {th }}$ edn), Tata McGraw-Hill, Hyderabad, India.

22. Zotin Al, Zotina RS (1967) Thermodynamic aspects of developmental biology. J Theor Biol 17: 57-75.

23. Baldwin JM (1896) A new factor in evolution. The American Naturalist 30 441-451.

24. Waddington $\mathrm{CH}$ (1953) Genetic assimilation of an acquired character. Evolution 7: 118-126.

25. Turner KH, Vallet-Gely I, Dove SL (2009) Epigenetic control of virulence gene expression in Pseudomonas aeruginosa by a LysR-type transcription regulator. PLoS Genet 5: 1000779. 